\title{
Gamemunication: Prosthetic Communication Ethnography of Game Avatars
}

\author{
SF. LUTHFIE ARGUBY PURNOMO \\ SF. LUKFIANKA SANJAYA PURNAMA \\ LILIK UNTARI \\ NUR ASIYAH \\ NOVIANNI ANGGRAINI \\ IAIN Surakarta, Indonesia
}

\begin{abstract}
This study revisits Hymes' ethnography of communication for game avatars, functioning as a communication nexus connecting games and gamers. Hymes formulates his ethnography of communication into SPEAKING (Settings and Scenes, Participants, Ends, Act Sequences, Keys, Instrumentalities, Norms, and Genres) and this formula deems to be unfit to explain how game avatars communicate. Implementing Klevjer's prosthetic telepresence (2012) to analyze sixty-two game titles, it is revealed that SPEAKING requires an extension when applied to study game avatars since the formula is not designed to explain the prosthetic nature of game avatars. This prosthetic nature produces specific communication ethnography of avatars, which we dub prosthetic communication ethnography. By prosthetic communication ethnography refers to technical elements of gaming, which contribute to the ways the avatars communicate. As Hymes' ethnography of communication with SPEAKING, this avatar based communication ethnography requires the same tool of analysis, which we call GAMING (Gaming systems, Attributes, Mechanics, Indexicalities, Narratives, and Geosocial systems), constructed with indexical storytelling by Fernández-Vara (2011), user interface types of games by Stonehouse (2014) and prosthetic video game theory by Jagodzinski (2019) as the theoretical foundations. GAMING and SPEAKING are integrated by bridging them with Aarseth's ludonarrative dimensions (2012).
\end{abstract}

Keywords: Game avatars, ethnography of communication, prosthetic, SPEAKING, GAMING.

\section{INTRODUCTION}

Avatar etymologically roots from Sanskrit word avatāra with ava meaning 'down' and tāra 'he crosses over' (Partridge, 2006). This etymology suggests a structural motion to perform the process of crossing over that involves a participant, a direction of crossing over, and a shift of place. In video game context, departing from this etymological meaning, the gamers are crossing over from their world to the game world, migration as Mustafa (2014) says, involving the gamers with their representations, the games and game machines as the means of crossing over and signifiers for a shift of place, a shift of world, and a shift of culture. This structural relationship gamers and avatars show is that of prosthetic relationship, a relationship resulting from the process of learning and habituation allowing the avatars as the extension of the gamers (Klevjer, 2006, 2012). This prosthetic relationship evokes an understanding that gamers and game avatars ethnographically employ particular means of communication in transferring information. Hymes' ethnography of communication, formulated into SPEAKING, is only able to explain how gamers interact in their speech community by analyzing the settings and scenes, participants, ends, act sequences, keys, instrumentalities, norms, and genres. Hymes' SPEAKING is unfit to analyze communication ethnography of game avatars since they are a part of the gamers but they exclude themselves 
from the gamers' speech community. In Multiplayer Online Battle Arena (MOBA) like Bizzard's World of Warcraft (WOW), Moonton's Mobile Legends, and Garena, Timi Studios, and Tencent Games' Arena of Valor (AOV), for instance, gamers are required to communicate with fellow gamers of the same team to defeat the opponent team and thus, MOBA related registers and team specific registers would dominate the communication in the context of cooperation and competition. Yet their communication is inseparable from interacting with game avatars. Gamers are required to comprehend the avatars not only their appearances but also their statistical attributes and motions. This attempt to comprehend avatars indicates that a process of communication between gamers and game avatars is taking place. This condition implies that game avatars have elements of communication, from which gamers decipher and signify the information those elements are trying to convey. Further, these elements of communication point that game avatars have their own unique ways to communicate with the gamers as their creators and controllers. This article attempts to prove the presence of these unique ways of communication by game avatars, which we call prosthetic ethnography of communication.

The issues of communication ethnography of avatars have been addressed by scholars from various backgrounds. Studies that address this issue are classifiable into those perceiving game avatars as a part of their gamers, those with opinions that game avatars should be treated as game avatars excluding the roles of gamers, and those who focus on the connection between game avatars and their gamers. Autoethnography, for instance, which focuses on the gamers and gaming community as the axis of communication with game avatars is discussed by the scholars of ethnography like Dumitrica and Gaden (2009), Downey (2012), and Shaw (2013) who hold the first perspective. Rehak (2003) and Giddings (2009) through their microethnographic perspectives attempts to elucidate how game avatars have a life of their own, from which the communication they perform is unfit to be interconnectively seen from the perspectives of gamers and their community. To compromise the two perspectives, the last perspective focusing on the information transfer based meaning making process between game avatars and their gamers is exercised. McArthur and Jenson (2015) and McArthur (2019) propose micro-autoethnography to address this issue. That game avatars are the prostheses of their gamers is the gap not yet explored. Perceiving game avatars as prostheses in the context of ethnography indicates that their communication ethnography is explorable with or without the presence of the gamers. This condition implies that the meaning signified from the communication process would be different, depending on whether the prostheses, game avatars, are attached to the gamers or detached out of them.

\section{Communication Ethnography of Game Avatars}

\section{LITERATURE REVIEW}

In the communications where game avatars, as the extension of the gamers, are the central focus, the cultural assumptions and manifestations are of prosthetic culture. In prosthetic telepresence (Klevjer, 2012), game avatars are the extension of gamer's presence and thus both are connected in a nexus of reciprocal communication. In regard to this nexus of reciprocal communication, avatars as the prosthetic engagement of the gamers situate a simulated circumstance for the gamers and the avatars themselves and thereby ethnography of communication, concerning on natural situation, erects a biased projection when applied for a simulated circumstance of video games. The biased projection is the result of treating avatars as the representation of gamers or treating them as beings with prosthetic cultures 
in a simulated world of video games. The first treatment of avatars indicates the necessity for a discussion on the cultural and contextual backgrounds of the gamers and the avatars. Executing this discussion, another bias occurs namely ludonarrative dissonance, a situation where in-game instruction contradicts the gameplay (Hocking, 2009), from which the avatars, at certain point of in-game time, are not in synchronization with the gamers.

The presence of ludonarrative dissonance as mentioned before implies that situated and simulated circumstances confuse the presence of interaction between the gamers and their avatars. The prosthetic relationship, in terms of communication, emphasizes that ludonarrative dissonance violates the structures of expectation, structures to which the interactants rely for a smooth communication (Ping \& Du, 2015). Analyzing ludonarrative dissonance from structures of expectation perspectives in ethnography of communication for a real life conversation denotes no conformity due to the absence of ludological traits in the analysis. The structures of expectation, in the context of video games, should be ludologically constructed. By ludological construction refers to the presence of branching interactions, which disclose opportunities for the gamers to approach the games from different perspectives and methods. In Yakuza series by Sega, for instance, the avatars are provided a simulated circumstance with usable and breakable objects for them to use when engaging in a battle. The presence of these usable and breakable objects to support the fight of the avatar indicates that the structure of expectation of the game is ludologically constructed to allow the avatars to access different perspectives and methods to win the fight. In the verbal context, structure of expectation in games also exposes a challenge of communication. In Konami's Metal Gear Solid, for instance, the gamers have to carefully examine the verbal textual hints to win a boss battle. When Snake has to battle Vulcan Raven, the game provides a verbal textual hint expressed by Raven in his dialogue "That's right. You belong on the ground. You should crawl on the ground like snake you are". These lines are a hint for the gamers to move Snake, the avatar, on the ground to avoid Raven's tank in order to win the battle. Supported by visual hints preceeding the emergence of verbal textual hints, the structure of expectation is designed as such to allow the gamers to win the battle as expected by the game mechanics.

This text signifies that SPEAKING formula should be prosthetically treated to fit to the ludological nature of the video games and thereby a derivational formula from SPEAKING is of necessity to be constructed to explain the ethnography of communication of video games. This claim is further supported by the fact that the communication avatars perform is of transdiegetic that merges communication from the game system with communication from the game world into a frame of reference that has usability value at the same time as it upholds the sense of presence in the game world (Jørgensen, 2008), from which the game avatars are convicted to have an influential communicative flow to other communicators while at the same time open for a communication with other communicators. This presence of communicators with avatars as the axis in video games indicates that avatars and those involving in a communication with them and their participations are parts of a structure. This participation in the context of prosthetic telepresence strengthens the necessity for game avatars as an ethnographic product to which ethnography of communication with distinctive features differentiating them from other ethnographic products to be structurally examined. Furthermore, the fact that the avatars are the prosthetic-self of the gamers indicates that in digital communication the ethnography of being communicators transdiegetically have nothing to do with the status of being an animate or an inanimate. 


\section{METHODOLOGY}

Seven procedures were taken in this descriptive qualitative research. First, data in the form of avatars were taken from sixty two game titles, which were selected purposively. Second, the avatars were classified based on Klevjer's prosthetic telepresence theory, Stonehouse's game interface theory, and Jagodzinski's prosthetic video game theory. Third, Klevjer's prosthetic telepresence theory was applied to examine the communication elements each avatar has and to reveal the presence of prosthetic communication ethnography. Fourth, revealing the prosthetic communication ethnography of game avatars, Hymes' like SPEAKING formula was constructed for a systemic and procedural steps of analysis with Aarseth's narrative theory and Fernández-Vara's indexical storytelling as the basis of construction. We call the formula GAMING (Gaming system, Attributes, Mechanics, Indexicalities, Narratives, and Geosocial systems). Fifth, Spradley's componential analysis theory (2016) was applied to reveal the relationship between the formula of prosthetic communication ethnography with the game avatars and the gamers. Sixth, the procedures on how to combine SPEAKING with GAMING in tandem were formulated. Seventh, an example of game avatar communication analysis was provided.

\section{RESULTS}

\section{Prosthetic Communication Ethnography of Game Avatars}

Investigating sixty two game titles, it was revealed that Hymes' SPEAKING demands a tandem with GAMING to address the prosthetic nature of game avatars. The prostheses game avatars have as a nexus of communication to the gamers comprise system, audiovisual, kinetic, onomastic, story, and social interaction prostheses. These prostheses are the elements of communication from which game avatars communicate with their gamers. Hymes' SPEAKING does not address these prostheses as a part of communication ethnography and thereby employing SPEAKING for an analysis on game avatar communication will end up in treating the way game avatars communicate the same as the way humans communicate. Therefore, a formula constructed from these six prostheses is required to explain how game avatars communicate and we call this formula GAMING, an acronym for Gaming systems, Attributes, Mechanics, Indexicalities, Narratives, and Geosocial systems.

\section{Prosthetic Game Avatars}

Drawing on Klevjer's prosthetic telepresence (2012), signifying the prosthetic nature of game avatars, Stonehouse's interface types (2014), indicating how gamers communicate with game world via user interface, and Jagodzinski's prosthetic video games (2019), highlighting how video games transform into a prosthetic learning where restriction on codes determines the learning process of gamers, a classification of what we call as prosthetic game avatars is constructed. Prosthetic game avatars are avatars which become the cyber extension and nexus of their gamers in mimetic, diegetic, and prosthetic communicative manners. Mimetic manners refer to the function of game avatars as unconscious or conscious representation of the gamers. Diegetic manners refer to the narratives game avatars have, from which the gamers through their game avatars have to explore. Prosthetic communicative manners are either verbal or metacommunication delivered by the game avatars, to which the gamers have to respond via controlling devices like console controllers, PC keyboards, and other controlling devices. 
We classify prosthetic game avatars into gamer-centric, game-centric, and gamingcentric avatars. Gamer-centric avatars are avatars created through character creation tools or features either provided by the games or through mods. These avatars aim at immersing the gamers as the center of the game narratives. To strengthen this gamer-centricism in the narratives, first person views are customarily provided as a viewpoint selection out of the widely used third person perspectives. Open world or sandbox games like Bethesda's Elder Scroll V: Skyrim and Bioware's Dragon Age Inquisition have this avatar type. Multiplayer games like Square Enix's Final Fantasy XIV Real Reborn or other game genres with role playing games (RPG) and online multiplayer elements and features like Soul Calibur VI and Dragon Ball Xenoverse series, though presented on third person and limited first person viewpoints, typically have this type of avatar also. Besides first person option, the use of diegetic or metadiegetic interface, in which the interface is not displayed on the screen or implicitly blended with the game environments, serves also as the characteristics of gamer-centric avatars. Ubisoft's Far Cry 5 is one of the games which diegetic and metadiegetic interface. The game provides the gamers the options to toggle off the interface menu to individualize the gaming experiences deeper. This individualizing immersion is the gaming value of gamercentric avatars.

Meanwhile game-centric avatars are avatars customized through customization tools or mods. This type of game avatar allows the gamers to do some modification on limited parts of the avatars, commonly on growth attributes and paraphernalia elements like costumes and equipments. Game-centric avatar dictates the gamers on how to interact with the game. Thus, gamers tend to function as the prosthesis of the game whereas gamer-centric avatars tend to function the way around. Since game-centric avatars tend to dictate the gamers, the interface used is non-diegetic and spatial, in which icons and symbols are utilized to tell the gamers what to do. The level of individualizing experiences this game avatar type provides is not as immersive as that of gamer-centric avatar. Nearly any game genres from side scrolling games like Capcom's Megaman series to RPG like Square Enix's Final Fantasy series have this type of avatar.

The last prosthetic avatar types, gaming-centric avatars, are not categorizable by the first two types. The word 'gaming' on this avatar refers to the gaming environment, in which the games separate between avatars serving as gamer identity and avatars as gamer's playable character. Games which provide offline and online features like Bandai Namco's Dragon Ball Fighter Z, for example, require gamers to create an avatar to traverse what is called as lobby, a place where gamers could interact with other players. Whilst gamer-centric and game-centric avatars are played to complete a game, gaming-centric avatars are not played in to complete a game but played to communicate with other gamers or nonplayable characters (NPCs). Gaming-centric avatars act as prosthesis to enhance the gaming skills of the gamers by sharing and learning tips and tricks from other gamers through online chat, competition, and cooperation.

\section{Gaming System and the Influences on the Communication of Prosthetic Game Avatars}

Gaming system refers to devices where gamers play their games. Gaming systems have their differences in presenting the games played. The same game titles might have different audiovisual and kinetic displays when played on different gaming systems. In game avatar context, gaming systems influence game avatars and how they communicate. Two types of 
influence appear from gaming systems toward game avatars namely intrinsic and extrinsic elements.

Intrinsic elements encompass any technical aspects gaming systems have e.g. CPU, GPU, storage, and other hardware and software specs. Konami's Suikoden series, for example, are played on different Playstation generations. The first and second series are Playstation games while the third, fourth, and fifth series are Playstation 2 games. Due to different specs, the avatars have different audiovisuals and functionalities. Suikoden and Suikoden II are played on 32 bit Playstation and thus 2D sprite is used to visualize the game avatars. Meanwhile Suikoden III, IV, and $V$ are 128 bit Playstation 2 games, polygonal graphics replace the 2D sprite. Even for the fourth and fifth series, avatars out of the main protagonists are voiced over. Though Suikoden // have more inferior graphics compared to its successors, it has the largest rosters of playable game avatars, implying that superiority in visual graphics sacrifices some aspects in functionalities. In the context of prosthetic avatars, all of the series have the same game-centric avatars with different range of customization options, indicating a difference scope of communication.

On the other hand, extrinsic elements refer to influences generated by contents in the forms of software, program, or application operated through the use of particular gaming systems and by noncontents like remakes or remasters. Extra contents like downloadable contents (DLCs), for instance, might drastically change the already existent game avatars and thus influencing the avatar appearance and even its statistical attributes. Most RPGs played for seventh and eight gen home consoles have avatar customization related avatar DLCs. The presence of these extra content indicates a metacommunication between the gamers and the DLCs, informing them regarding with the prostheses which might benefit the gamers in exploring the games. Thus, selecting the DLCs to modify the avatars signifies the prosthesis status of the DLCs. Besides DLCs, mods are also a popular content which might influence game avatars. Games with self-development kit (SDK) like Grand Theft Auto series disclose a wide range of possibilities for gamers to modify any contents of the games as they please, including the avatars.

Extrinsic influences are not only generated by contents but also noncontents. The latter refers to what Klein and Palmer (2016) call as multiplicities, intertextual products derived from an existing product which might take the forms of remakes, remasters, reboots, sequels, prequels, and spin-offs. These multiplicities, as what extra contents do, might influence game avatars. When games like Bandai Namco's Tales of Innocence, Tales of Vesperia, and Tales of Graces are remade for the same or different gaming systems from the original, they introduce new characters playable by the gamers as avatars and thus experiences called as commodified nostalgia (Cuff \& Terry, 2017) are obtainable by new and veteran players.

\section{Attributes and the Growth of Communication Ability of the Prosthetic Game Avatars}

Attributes refer to statistical attributes which determine the avatar growth. The communication attributes of the avatars attempt to make tends to be calculative and logic since gamers are provided by mathematical like equations, from which the gamers make decisions regarding with the game avatars. Game avatars gain statistical attributes from actions, objects, and mods. RPG titles have statistical attributes in the form of what is called as experience point (EXP). These EXPs are obtainable from performing particular actions like defeating enemies and completing missions. EXPs are also acquired through equipping game avatars with particular equipment or consuming certain items. Modification by utilizing 
particular cheating tools like Game Shark or modding kits might overwhelmingly boost one's game avatars' statistical attributes.

The presence of these three methods suggests that a metacommunication is occuring. Once gamers comprehend that defeating an enemy has a direct impact on the growth of their game avatars, gamers especially in RPG titles would do what is conventionally termed as level grinding. This type of action is done by spending some time to do an action of defeating as many enemies as possible to boost the statistical attributes of the game avatars. This level grinding is customarily exercised when the gamers need to defeat powerful enemies conventionally dubbed as bosses. These level grinding actions are rarely explicitly suggested by the games. Gamers metacommunicatively comprehend these actions through the comprehension of statistical attribute comparison between their game avatars and the bosses they attempt to engage. This comprehension indicates that statistical attributes are the prosthesis game avatars have, from which the gamers use them as a guide to make a decision.

In the context of prosthetic game avatars, gamer-centric and game-centric avatars share similar metacommunication on statistical attributes. Differences on the methods to gain the attributes are not linked to the avatar types but to the game mechanisms. Meanwhile gaming-centric avatars have a quite different function of statistical attributes. If gamer-centric and game-centric avatars benefit the attributes for their growth, from which the gamers might take particular advantages in completing the games, gaming-centric avatars tend to benefit from the attributes for recognition purposes. In games with online features like the aforementioned Bandai Namco's Dragon Ball Fighter Z, for example, gamers from around the world fight each other through Bandai Namco's servers. When they win or lose, their gamingcentric avatar's names appear on what is called as leaderboard, a ranking board containing the data of all online players and their online achievements. Winning a game gains the gamers statistical attributes and vice versa for losing a game. These statistical attributes determine the ranking position on the leaderboard and thus, as suggested by King, Delfabbro, and Griffiths (2010), encouraging social competition among gamers. This social competition indicates that game avatars are utilized as social prostheses by the gamers to achieve a social recognition.

The Mechanics and the Kinesics of Communication of the Prosthetic Game Avatars

The third ethnographic communication element game avatars have is mechanics, which concerns on the kinetic aspects of prosthetic game avatars. Kinesics in game avatar context refers to motions and actions of game avatars in exploring the game environment and narrative. Kinesics, just like humans do with gestures, is a communication tool of the game avatars in delivering information to the gamers (Manninen, 2003).

Departing from how the communication is exercised, we classify kinesics into protokinesic, merokinesics, and sociokinesics. The first refers to motions and actions which mimetically visualize synchronicity between the motions and actions of the gamers with the motions and actions of the game avatars. In protokinesics, the original motions and actions of the gamers are visually mimicked by the game avatars. Wii games like Wii Sports enable this synchronicity through the use of wiimote or nunchuk controllers. Swinging the controllers, for instance, the gamers will notice that the gamer avatars will do the same action. This type of kinesics attempts at immersing gamers as one with their game avatars in the game world and thereby, gamer-centric prosthetic avatars tend to fit this kinesics type. 
The second kinesics type, merokinesics, refers to the motions and actions which focus not on mimetic synchronicity but diegetic synchronicity, implying that any movements are delivered through button pressing. This is the most common type of kinesics implemented on games. Games with the three types of prosthetic game avatars employ this kinesics types with differences on the button functions and controller types.

The last kinesics type, sociokinesics, occurs in a virtual reality (VR) games or games with VR modes. In Bandai Namco's Ace Combat 7: The Unknown Skies, for instance, the gamers totally immerse themselves as a pilot in the game. Any flight related experiences like navigation, battle, and communication with bases are kinetically responded by the gamers in a virtual environment. Motions and actions are not the only kinetic elements mimetically visualized by the games but also visual perceptions through the use of VR goggles. This last kinesics suggests that the gamers are a part of the in-game society, in which interactions between the game characters and the gamers are virtually real.

\section{Indexicalities and Identities of Communication of the Prosthetic Game Avatars}

Storytelling in games is basically indexical storytelling, in which any textual, audial, visual, and kinetic elements on the games are designed to generate hints or clues for the gamers to respond (Fernández-Vara, 2011). These indexicalities imply that textual, audial, visual, and kinetic elements of a game are the prostheses the game has in making a communication with the gamers. In game avatar context, textual elements are indexically realized through onomastic aspects, the naming of the avatars. The names game avatars bear reflects the identity of the gamers. Supported by visualization, as an inseparable part of meaning making element (Sjafiie, Hastjarjo, Muktiyo \& Pawito, 2018), the names become the point of departure in signifying the game avatars. We classify game avatar naming into respective, reflective, and representative. Respective naming disallows gamers to alter the already existing names provided by the games, reflective naming allows an alteration, and representative naming demands the gamers to create a name for the game avatars. Each of this naming type generates a different channel of identity, in which in animated character context identity must be narrated along with personality (Khalis \& Mustaffa, 2017), and index based meaning making process.

The identities respective naming attempts to communicate are narrative bound, implying that game-centricism is the primary concern. Since game-centricism is the essential element of respective naming, gamers tend to be the prostheses of the games. The indexical communication under this respective naming type indicates that the unalterable names given to the avatars are static. This static communication implies that the meaning the names as textual elements, the body as visual, the voices as audial, and the motions and actions as kinetic attempt to weave is already predetermined. The meaning might be predetermined but the deciphering process is dynamic since the indices are implicitly embedded to the games, lying as dormant information, and gamers are required to capture and decipher it.

The counterpart of this naming is representative naming, in which gamer-centricism is the main focus. In this gamer-centric avatar related naming classification, gamers are the center. After an avatar is physically created, an onomastic identity for the avatar comes next. Representative naming frees gamers to accomplish this onomastics related action and this freedom complicates the indexicalities the game avatars attempt to build. Audial, visual, and kinetic aspects of the game avatars are subjectively linked to the names of the avatars and thus, the meaning the aspects attempt to transfer might be detachable from the game narratives. Square Enix's Dragon Quest series, for instance, have their main heroes in a game- 
centric prosthetic avatar fashion but freeing gamers to name the avatar. If an aesthetics analysis on game narrative with onomastics as the point of departure is taken, difficulties arise in connecting the name meaning with the overall intrinsic storytelling elements of the games as indices to decipher a hidden meaning of the narrative.

A quite different concern is given from the second naming classification, reflective naming. In this naming, narrative and personal identities are negotiated, leaving gamecentricism and gamer-centricism in between with a focus given more on gaming identity. This negotiation implies the existence of what Hassan and Novchi (2019) address as socioculturality considerations, where nuance and balance of personal and sociocultural aspects are accomodated. In Square Enix's Final Fantasy $X$, gamers control the main protagonist called Tidus. The name is strongly linked to the ocean theme the game attempts to tell. This theme linked connection might suffer a narrative distortion since the name 'Tidus' is alterable.

\section{Narratives and Stories as a Means of Communication for Prosthetic Game Avatars}

Narratives in GAMING context concern on the abilities of game avatars in exploring game narratives. We classify these abilities based on the freedom of narrative exploration by game avatars. Borrowing literary terms, they are called sylleptic, proleptic, and metaleptic narratives. Sylleptic narratives are narratives of which the freedom of exploration is explicitly presented in a textual pathway branching. Visual novel game genres like D3P and Red Entertainment's Nightshade and Capcom's Phoenix Wright series, for example, commonly have this narrative exploration type. Gamers are presented by textual choices called pathways which branch out the narratives, indicating the presence of nonlinear narrative, presenting events in a nonlinear time sequence (Hashim, 2019). Game-centric avatars commonly inhabit this narrative type.

Proleptic narratives are defined the freedom to perceive the game narratives through multiple perspectives of different game avatars, from which a particular narrative is set as a meeting point. Konami's Suikoden III, for example, has Trinity system, allowing the gamers to experience the game main narrative from three different narratives plus one extra narrative. Similar approach is also taken by Square Enix's Octopath Traveler. As the title suggests, gamers control eight game-centric avatars by having one at the start and as the story flows, gamers will be able to gather all the eight. This narrative type prompts gamer's curiosity over how other characters see and respond toward a single event in the narratives.

Metaleptic narratives mostly deal with total freedom in exploring game narratives by gamer-centric prosthetic avatars. Games like Rockstar's Grand Theft Auto and Red Dead Redemption series are unbound by narrative limitation, pointing out the freedom gamers have in exploring the game narratives from any points of departure. This narrative exploration freedom indicates that game narratives are prosthetically designed in a gamer-centricism paradigm, from which game narratives are explorable in a personal perspective.

\section{Geosocial Systems and the Social Communication of Prosthetic Game Avatars}

Geosocial systems deal with physical and physiological elements of game avatars, from which social communication related meaning making processes occurs. Though game avatar creation tools or features disclose limitless possibilities in the results, they are socially attached to game avatars-game and game avatars-game avatars communication. Departing 
from this social communication, geosocial systems are classified into gnomic and genomic geosocial systems.

Gnomic refers to jobs or classes of the game avatars, from which other game-avatars might take advantage from. Massively Multiplayer Online Role Playing Games (MMORPGs) like Gravity's Ragnarok Online series have this job or class system. If a gamer chooses his avatar the job mage or magician, it indicates that the avatar is useful for long ranged combat. Capturing this indexical message, other gamers through their game avatars could ask the mage to join their party if the party lacks of a long range combat specialist like mage. This social interaction indicates that jobs or classes socio-communicate the avatars to other gamers via their game avatars. If the mage agrees to join the party, it points out that gnomic geosocial system enters the status of genomic geosocial system.

Genomic refers to social communication shared by game avatars gathering in a collective group and sharing the same purpose. In MMORPG context, this collective group is called guild. Once game avatars are in a guild, they will work together to make other guilds and any game avatars in the game environment recognize them. This recognition shared as an end by the guild is achieved through battles against other guilds in a war called guild war. Emerging victorious, their rank and fame will rise. The process of achieving this end signifies the presence of an intensive social communication between game avatars of the same guild via their game avatars. In prosthetic communication context, the existence of guild indicates that game avatars are utilized as the social prostheses of the guild to achieve a common goal.

\section{DISCUSSION}

Gnomic and genomic geosocial systems evoke an understanding that social communication performed by game avatars might be transformatively linked. In this geosocial systems, game avatars are impossible to embrace genomic status if they have not been gnomically created. These geosocial systems also spark an interesting question regarding with prosthetic gameavatar classifications. Does the status as gamer-centric, game-centric, or gaming-centric avatars alter when game avatars enter gnomic and genomic geosocial systems? Fundamentally the classification of prosthetic game avatars is seen from whether they are created or customized, whether they are functioned to explore a game world or a transitional place before exploring the game world, and whether they are dominantly presented through particular interface. The following table might help illustrate these elements of classification:

Table 1: Prosthetic game avatars classification

\begin{tabular}{|c|c|c|c|c|c|c|c|c|}
\hline & \multicolumn{2}{|c|}{ Initiation } & \multicolumn{2}{|c|}{ Event } & \multicolumn{4}{|c|}{ Interface } \\
\hline & Creation & $\begin{array}{c}\text { Customiza- } \\
\text { tion }\end{array}$ & $\begin{array}{c}\text { Non- } \\
\text { Transitional }\end{array}$ & Transitional & Diegetic & $\begin{array}{c}\text { Metadiege } \\
\text {-tic }\end{array}$ & $\begin{array}{c}\text { Non- } \\
\text { diegetic }\end{array}$ & $\begin{array}{l}\text { Spa- } \\
\text { tial }\end{array}$ \\
\hline $\begin{array}{l}\text { Gamer- } \\
\text { Centric }\end{array}$ & V & $x$ & $\mathrm{~V}$ & $x$ & V & $\sqrt{ }$ & $x$ & $x$ \\
\hline $\begin{array}{l}\text { Game- } \\
\text { Centric }\end{array}$ & $x$ & v & $\sqrt{ }$ & $x$ & $x$ & $x$ & v & $\sqrt{ }$ \\
\hline $\begin{array}{l}\text { Gaming- } \\
\text { Centric }\end{array}$ & $x$ & v & $x$ & v & $x$ & $x$ & $\sqrt{ }$ & $\sqrt{ }$ \\
\hline
\end{tabular}

As seen from the table, game avatars are classified into gamer-centric when the avatars are initiated by creation, designed to engage in a non-transitional exploration, and immersed in a diegetic and metadiegetic interface. These elements embedded on gamercentric avatars indicate that initiation, event, and interface elements are utilized as the prostheses for the game avatars, from which a personal and individualizing immersion is the 
primary objective of the avatar design. Meanwhile game-centric and gaming-centric avatars tend to share similar elements of classification with non-transitional and transitional events as the only difference. These similarities indicate that both avatars tend to function the classification elements as the prostheses of the games attached to the game avatars. Departing from this understanding, prostheses in game avatar context are mainly categorized into game-to-gamer and gamer-to-game prostheses.

Game-to-gamer prostheses are prostheses which attach the functionality of classification elements the games provide to gamers and thus making the game avatars tend to be gamer-centric whereas gamer-to-game prostheses work vice versa. The following table provides the relationship between prostheses, GAMING, and prosthetic game avatars:

Table 2: Prostheses, GAMING, and prosthetic game avatars

\begin{tabular}{|c|c|c|c|c|c|c|c|c|c|c|c|c|c|c|c|}
\hline & \multicolumn{2}{|c|}{$\begin{array}{l}\text { Gaming } \\
\text { Systems }\end{array}$} & \multicolumn{3}{|c|}{ Attributes } & \multicolumn{3}{|c|}{ Mechanics } & \multicolumn{3}{|c|}{ Indexicalities } & \multicolumn{3}{|c|}{ Narratives } & \multirow{2}{*}{$\begin{array}{l}\text { Geosocial } \\
\text { Systems } \\
\text { Gno Gen }\end{array}$} \\
\hline & Int & Ext & Act & Obj & Mod & Proto & Mero & Socio & Res & Ref & Rep & Syl & Pro & Met & \\
\hline $\begin{array}{l}\text { Gamer- } \\
\text { Centric }\end{array}$ & Gtg & Gtg & Gtg & Gtg & Gtg & Gtg & Gtg & Gtg & $x$ & $x$ & Gtg & Gtg & Gtg & Gtg & Gtg Gtg \\
\hline $\begin{array}{l}\text { Game- } \\
\text { Centric }\end{array}$ & gtG & gtG & gtG & gtG & gtG & $x$ & gtG & $x$ & gtG & $x$ & $x$ & gtG & gtG & gtG & gtG gtG \\
\hline $\begin{array}{l}\text { Gaming- } \\
\text { Centric }\end{array}$ & gtG & gtG & gtG & gtG & gtG & $x$ & gtG & $x$ & $x$ & gtG & $x$ & gtG & gtG & gtG & gtG gtG \\
\hline
\end{tabular}

Gtg: Game-to-gamer, gtG: gamer-to-Game, Int: internal influences, Ext: external influences, Act: action, Obj: object, Mod: modding, Proto: protokinesics, Mero: merokinesics, Socio: sociokinesics, Res: respective, Ref: reflective, Rep: representative, Syl: sylleptic, Pro: proleptic, Met: metaleptic, Gno: gnomic, Gen: genomic

As seen from the table, gamer-to-Game dominates prosthetic game avatars. This dominant finding indicates that gamer-centric avatars have the most complete communication elements. Though gamer-centric avatars are the avatars with the most complete communication elements, they share the same domain as game-centric and gaming-centric avatars in what is called as ludonarrative dimensions, a meeting point of narrative and mechanical aspects of games (Aarseth, 2012). Ludonnarative dimensions are applicable to connect Hymes' SPEAKING and GAMING by treating GAMING as the representation of game's mechanical aspects and SPEAKING as that of narrative aspects.

Ludonarrative dimensions, as Aarseth (2012) claims, have four dimensions namely worlds, objects, agents, and events. The following table provides the sub classification of each dimension:

Table 3: Ludonarrative dimensions

\begin{tabular}{cccc}
\hline Worlds & Objects & Agents & Events \\
\hline Linear & Dynamic & Rich character & Open \\
Multicursal & User created & Deep character & Selectable \\
Open & Static & Round character & Plotted \\
\hline
\end{tabular}

Employing Aarseth's ludonarrative dimension model to bridge GAMING and SPEAKING implies the necessity to adjust the elements of both communication ethnography formulae to the dimensions. The following table might help how GAMING and SPEAKING elements are made into tandem with ludonarrative dimensions as the bridge. 


\begin{tabular}{|c|c|c|}
\hline & GAMING & SPEAKING \\
\hline \multirow[t]{2}{*}{ Worlds } & Geosocial systems & Settings \\
\hline & & Scenes \\
\hline Objects & Gaming systems & Instrumentalities \\
\hline \multirow[t]{2}{*}{ Agents } & Attributes & Participants \\
\hline & Mechanics & \\
\hline \multirow[t]{5}{*}{ Events } & Narratives & Act Sequences \\
\hline & Indexicalities & Ends \\
\hline & & Keys \\
\hline & & Norms \\
\hline & & Genres \\
\hline
\end{tabular}

Departing from the fusion, as seen from the table above, prosthetic communication ethnography of game avatars is able to be analyzed. Dragonborn of Bethesda's Elder Scroll V: Skyrim, for instance, is classified into gamer-centric since the avatar fulfills two of three requirements to be called as gamer-centric avatar namely initiation through creation system and direct game world exploration. The world where Dragonborn has to explore, Skyrim, is open. The openness of the world is supported by gnomic geosocial system which frees the gamers in developing the Dragonborn's skills though point distribution. The openess is also supported by the freedom to explore the various settings of Skyrim from isolated forests into densely populated cities and the freedom to do various acts which might trigger various physical or psychological traits driven scenes. Objects, agents, and events are also aligned to support this openness of Skyrim, where every action matters. Playstation 3 specs and Bethesda's Creation Engine make this openness possible, indicating that every game element presented in the game acts as prosthesis for the gamers to achieve personal and individualizing immersive experiences.

In the perspectives of information models, these immersive experiences are communicated in regard to how game information is delivered to gamers through game avatars. Two information models namely adhoc and hybrid adhoc models occur. The former refers to purpose based communication while the second to a combination between purpose and centralization based communication models (Kasapakis \& Gavalas, 2015). In relation to how game avatars contribute to the immersive experiences of the gamers, adhoc and hybrid adhoc have different scopes of gaming experience immersion. In multiplayer or team based e-sports competitions, for example, hybrid adhoc information models are primarily employed to transfer strategizing based information with centralized command of the team's captain. In regard to game avatars, the immersion experienced by gamers tends to be narrative immersion, where reliance on mental activity becomes the essence of the experience (Ryan, 2015).

Since mental activities are the core of immersive experiences, intimate communication Ensslin (2011) calls as 'buddylect', a highly encoded and personalised communication comprehended only by buddies or team members, is the communication type e-sport team members engage to. The presence of buddylect indicates that the mental activities operate on a specific manner of cognition, allowing an efficient way of communication. Lipovaya et al. (2018) call this specific cognition team cognition, from which anticipation and prediction are the cores of communication between team members. In 
regard to game avatars, it implies that prosthetic communication is operated through the efficient use of game avatars as a tool of cognition.

\section{CONCLUSION}

Hymes' SPEAKING, which stands for Settings and Scenes, Participants, Ends, Act Sequences, Keys, Instrumentalities, Norms, and Genres, requires a tandem when applied to analyze game avatars since the formula is not intended to examine and explore the prosthetic nature of games. This article proposes GAMING which stands for Gaming systems, Attributes, Mechanics, Indexicalities, Narratives, and Geosocial systems. SPEAKING and GAMING are fusible by employing Aarseth's ludonarrative dimensions namely worlds, objects, agents, and events. Each element of SPEAKING and GAMING formula is classified under the four dimensions to reveal how GAMING operates around mechanical dimensions, to which the word 'ludo' refers, and how SPEAKING operates around narrative dimensions, to which the word 'narra' refers.

SPEAKING and GAMING integration starts from revealing game avatar types. We propose three types of game avatars namely gamer-centric, game-centric, and gamingcentric. These three avatar types are differentiated through their initiations, events, and interfaces. Departing from these classification elements, protheses which become the main focus are recognizable namely game-to-gamer ( $\mathrm{Gtg}$ ) and gamer-to-game (gtG) prostheses. The former prosthesis is found from gamer-centric avatars, designed to personalize and individualize the immersive experiences of the gamers. The latter is found from game-centric and gaming-centric avatars, designed to immerse the players in a procedural mechanism the games set.

The integration of SPEAKING and GAMING formula with prosthetic game avatars as the axis suggests the presence of what we call as prosthetic communication ethnography. This ethnography aims at revealing how gamers and game avatars communicate for a mutual communication exchange by treating games as prostheses which connect game avatars and their gamers. Game designers might benefit this ethnography when designing a game in relation to the gaming experiences the designed games attempt to generate. Linguists might also take this ethnography to analyze how gamers and game avatars communicate in a specific gaming related context like e-sports. 


\section{BIODATA}

SF. Luthfie Arguby Purnomo is a lecturer at English Letters Department, Faculty of Language and Culture, IAIN Surakarta. His research interests are in audiovisual translation, video game translation, game localization, game studies, and ludic linguistics. Email: theluthfie@gmail.com

SF. Lukfianka Sanjaya Purnama is a lecturer at English Letters Department, Faculty of Language and Culture, IAIN Surakarta. His research interests are in audiovisual translation, video game translation, game localization, game studies, ludic linguistics, and popular culture. Email: iaftersmile@gmail.com

Lilik Untari is a senior lecturer at English Letters Department, Faculty of Language and Culture, IAIN Surakarta. Her research interests are in translation, audiovisual translation, video game translation, and linguistics. Email: nazala.suha@gmail.com

Nur Asiyah is a lecturer at English Letters Department, Faculty of Language and Culture, IAIN Surakarta. Her research interests are in audiovisual translation, literary studies, and American studies. Email: assyong2@gmail.com

Novianni Anggraini is a lecturer at English Language Education Dept, Faculty of Language and Culture, IAIN Surakarta. Her research interests are in language and education, translation, and educational technology. Email: beinghappymom@gmail.com. 


\section{REFERENCES}

Aarseth, E. (2012, May). A narrative theory of games. Proceedings of the international conference on the foundations of digital Games, 129-133. ACM.

Cuff, S., \& Terry, C. (2017). Commodified nostalgia and the virtual console. The Evolution and Social Impact of Video Game Economics, 15.

Downey, G. (2012). The here and there of a femme cave: An autoethnographic snapshot of a contextualized girl gamer space. Cultural Studies? Critical Methodologies, 12(3), 235241.

Dumitrica, D., \& Gaden, G. (2009). Knee-high boots and six-pack abs: Autoethnographic reflections on gender and technology in Second Life. Journal for Virtual Worlds Research, 1(3).

Ensslin, A. (2011). The language of gaming. London: Macmillan International Higher Education.

Fernández-Vara, C. (2011). Game spaces speak volumes: Indexical storytelling. Proceedings of DiGRA 2011 Conference: Think Design Play.

Giddings, S. (2009). Events and collusions: A glossary for the microethnography of video game play. Games and Culture, 4(2), 144-157.

Hashim, H. (2019). Narrative Techno-enhancement: The Impact of the Digital Visual Effects DVFx) in Creative Narrative Performance. Jurnal Komunikasi: Malaysian Journal of Communication, 35(1).

Hassan, B. R. A., \& Novchi, R. W. (2019). Kepeminatan dan Kemodenan dalam Pembentukan Identiti Komuniti K-Popers Pekanbaru. Jurnal Komunikasi: Malaysian Journal of Communication, 35(1).

Hymes, D. (1962). The ethnography of speaking. Anthropology and human behavior, 13(53), 11-74.

Hocking, C. (2009, January). Ludonarrative dissonance in Bioshock: The problem of what the game is about. In Well Played 1.0 (pp. 114-117). ETC Press.

Jagodzinski, J. (2019). The prosthetics of video games. Schizoanalytic Ventures at the End of the World: Film, Video, Art, and Pedagogical Challenges, 149-165.

Jørgensen, K. (2008). Audio and gameplay: An analysis of PvP battlegrounds in World of Warcraft. Game Studies, 8(2).

Kasapakis, V., \& Gavalas, D. (2015). Pervasive gaming: Status, trends and design principles. Journal of Network and Computer Applications, 55, 213-236.

Khalis, F. M., \& Mustaffa, N. (2017). Cultural inspirations towards malaysian animation character design. Jurnal Komunikasi: Malaysian Journal of Communication, 33(1).

King, D., Delfabbro, P., \& Griffiths, M. (2010). Video game structural characteristics: A new psychological taxonomy. International journal of mental health and addiction, 8(1), 90-106.

Klevjer, R. (2006). What is the avatar? Fiction and embodiment in avatar-based single player computer games (Doctoral thesis, The University of Bergen, Norway). Retrieved from http://hdl.handle.net/1956/2234

Klevjer, R. (2012). Enter the avatar: The phenomenology of prosthetic telepresence in computer games. In The philosophy of computer games (pp. 17-38). Dordrecht: Springer.

Klein, A. A., \& Palmer, R. B. (Eds.). (2016). Cycles, sequels, spin-offs, remakes, and reboots: Multiplicities in film and television. Texas: University of Texas Press. 
Lipovaya, V., Lima, Y., Grillo, P., Barbosa, C. E., de Souza, J. M., \& Duarte, F. J. D. C. M. (2018). Coordination, communication, and competition in eSports: A comparative analysis of teams in two action games. Proceedings of 16th European Conference on ComputerSupported Cooperative Work-Exploratory Papers, European Society for Socially Embedded Technologies (EUSSET).

Manninen, T. (2003, May). Conceptual, communicative and pragmatic aspects of interaction forms-rich interaction model for collaborative virtual environments. Proceedings 11th IEEE International Workshop on Program Comprehension, 168-173.

Mcarthur, V., \& Jenson, J. (2015). Plans and co-situated factions: An evaluation of avatar affordances in Rift's character creation interface. Journal of Gaming \& Virtual Worlds, 7(1), 77-99.

McArthur, V. (2019). Making ourselves visible: Mobilizing micro-autoethnography in the study of self-representation and interface affordances. Loading: The Journal of the Canadian Game Studies Association, 12(19).

Partridge, E. (2006). Origins: A short etymological dictionary of modern English. UK: Routledge.

Ping, D., \& Du, P. (2015). Intercultural communication in the Chinese workplace. Berlin: Springer.

Rehak, B. (2003). Playing at being: The video game theory reader. NY: Routledge.

Ryan, M. L. (2015). Narrative as virtual reality 2: Revisiting immersion and interactivity in literature and electronic media (Vol. 2). US: JHU Press.

Shaw, A. (2013). Rethinking game studies: A case study approach to video game play and identification. Critical studies in media communication, 30(5), 347-361.

Siti Ezaleila Mustafa. (2014). Penghijrahan dari dunia realiti ke dunia maya: Tinjauan umum terhadap dunia maya sosial terpilih. Jurnal Komunikasi: Malaysian Journal of Communication, 30(Special Issue), 243-258.

Sjafiie, S. S. L., Hastjarjo, S., Muktiyo, W., \& Pawito. (2018). Graphic visualization in printed media: How does the use of technology influence journalism culture. Jurnal Komunikasi: Malaysian Journal of Communication, 34(4), 373-385.

Spradley, J. P. (2016). Participant observation. Illinois: Waveland Press.

Stonehouse, A. (2014). User interface design in video games. GAMASUTRA: The Art \& Business of Making Games. Retrieved on August 3, 2014, from https://www.gamasutra.com/blogs/AnthonyStonehouse/20140227/211823/User_in terface_design_in_video_games.php 